\title{
DEHN FILLING: A SURVEY
}

\author{
C. McA. GORDON \\ Department of Mathematics, The University of Texas at Austin \\ Austin, Texas 78712-1082, U.S.A. \\ E-mail: gordon@math.utexas.edu
}

1. Introduction. In this paper we give a brief survey of the present state of knowledge on exceptional Dehn fillings on 3-manifolds with torus boundary.

For our discussion, it is necessary to first give a quick overview of what is presently known, and what is conjectured, about the structure of 3-manifolds. This is done in Section 2. In Section 3 we summarize the known bounds on the distances between various kinds of exceptional Dehn fillings, and compare these with the distances that arise in known examples. In Section 4 we make some remarks on the special case of complements of knots in the 3 -sphere.

We have chosen to phrase questions as conjectures; this gives them a certain edge and perhaps increases the likelihood that someone will try to (dis)prove them. Incidentally, no particular claim is made for unattributed conjectures; most of them are lore to the appropriate folk.

Related survey articles are [Go1] and [Lu].

I would like to thank Pat Callahan, Craig Hodgson, John Luecke, Alan Reid and Eric Sedgwick for helpful conversations, and the referee for his useful comments.

2. 3-Manifolds. Throughout this section, all 3-manifolds will be closed and oriented.

Recall that the connected sum of two 3-manifolds $M_{1}$ and $M_{2}$ is defined by removing the interior of a 3-ball from each of $M_{1}$ and $M_{2}$ and identifying the resulting boundaries by an orientation-reversing homeomorphism. This gives a manifold $M_{1} \# M_{2}$, which is well-defined (as an oriented manifold). In particular, $M \# S^{3} \cong M$ for all $M$.

A 3-manifold is prime if $M \cong M_{1} \# M_{2}$ implies that either $M_{1}$ or $M_{2}$ is homeomorphic to $S^{3}$.

1991 Mathematics Subject Classification: 57M25, 57M50.

Partially supported by NSF Grant DMS-9303229.

The paper is in final form and no version of it will be published elsewhere. 
There is the following prime factorization theorem for 3-manifolds with respect to connected sum.

Theorem 2.1 (Kneser [K], Milnor [Mi2]). For every 3-manifold $M$ there exist prime 3-manifolds $M_{1}, \ldots, M_{n}$ such that $M \cong M_{1} \# \cdots \# M_{n}$. The summands $M_{i}$ not homeomorphic to $S^{3}$ are unique up to order.

It is convenient to say that $M$ is irreducible if every 2 -sphere in $M$ bounds a 3 -ball in $M$. Thus prime and irreducible are almost equivalent, the only exception being $S^{1} \times S^{2}$, which is prime but not irreducible.

In view of Theorem 2.1, it is enough to study irreducible 3-manifolds. For these, we have the following theorem.

TheOREM 2.2. Let $M$ be an irreducible 3-manifold. Then either

(1) $\pi_{1}(M)$ is finite; or

(2) $\pi_{1}(M)$ is infinite and $\pi_{i}(M)=0, i \geq 2$.

(Conversely, (1) and (2) each implies that $M$ is irreducible, modulo the Poincaré Conjecture.)

Part (2) is an easy consequence of the sphere theorem [Pap], [Wh].

Note that in case (1), the universal cover $\widetilde{M}$ of $M$ is compact and simply-connected, and hence a homotopy 3 -sphere, while in case $(2), \widetilde{M}$ is contractible. It is conjectured that $\widetilde{M}$ is homeomorphic to $S^{3}$ or $\mathbb{R}^{3}$, respectively.

We now describe the expected structure of 3-manifolds of types (1) and (2) in more detail.

(1) $\pi_{1}(M)$ finite. The conjectured picture here is quite simple. Let $S^{3}$ be the unit sphere in $\mathbb{R}^{4}$ with the metric induced by the euclidean metric on $\mathbb{R}^{4}$. The linear action of $O(4)$ on $\mathbb{R}^{4}$ restricts to an action on $S^{3}$, and in this way the group of orientation-preserving isometries of $S^{3}, \operatorname{Isom}^{+}\left(S^{3}\right)$, is identified with $S O(4)$. If $\pi$ is a finite subgroup of $S O(4)$ such that the action of $\pi$ on $S^{3}$ is free then the quotient $S^{3} / \pi$ is a 3-manifold $M$ with $\pi_{1}(M) \cong \pi$. Such a 3 -manifold $M$ is a 3 -dimensional spherical space form. One also says that $M$ is spherical or elliptic.

The groups $\pi$ of this form, and the corresponding 3-manifolds, are completely classified [Hop], [TS].

COnjeCture 2.3. Any 3-manifold $M$ with $\pi_{1}(M)$ finite is spherical.

By considering the universal cover of $M$ one may regard the above conjecture as being broken up into three parts:

(i) if $\pi_{1}(M)$ is trivial then $M \cong S^{3}$;

(ii) if $\pi$ acts freely on $S^{3}$ (or perhaps a homotopy 3 -sphere) then $\pi$ is isomorphic to a subgroup of $S O(4)$ whose restriction to $S^{3}$ is free;

(iii) if $\pi$ is a subgroup of $S O(4)$ whose restriction to $S^{3}$ is free, then any free action of $\pi$ on $S^{3}$ is conjugate to its action as a subgroup of $S O(4)$.

(i) is of course the Poincaré Conjecture.

(ii) is almost solved [Mi1], [Le].

(iii) is known for some groups $\pi$ [Li], [Ric], [Rit], [EM], [Ru], [My]. 
(2) $\pi_{1}(M)$ infinite, $\pi_{i}(M)=0, i \geq 2$. Here it turns out that it is important to distinguish two subcases.

(a) $\pi_{1}(M)$ has no subgroup isomorphic to $\mathbb{Z} \times \mathbb{Z}$. Examples of such manifolds arise as follows. Let $H^{3}$ be 3-dimensional hyperbolic space, in other words, one of the two components of the unit sphere in 4-dimensional Minkowski space. Using the upper halfspace model for $H^{3}$, the group $\operatorname{Isom}^{+}\left(H^{3}\right)$ of orientation-preserving isometries of $H^{3}$ can be identified with $\mathrm{PSL}_{2}(\mathbb{C})$, the group of linear fractional transformations of the Riemann sphere. If $\pi$ is a subgroup of $\operatorname{Isom}^{+}\left(H^{3}\right)$ whose action on $H^{3}$ is free and properly discontinuous and has compact quotient, then this quotient $M=H^{3} / \pi$ is a 3 -manifold with $\pi_{1}(M) \cong \pi$. Moreover, no such $\pi$ can have a subgroup isomorphic to $\mathbb{Z} \times \mathbb{Z}$. The manifold $M$ is said to be hyperbolic.

Seifert and Weber gave one of the first examples of such a manifold, the hyperbolic dodecahedral space, in [WS].

The central role played by hyperbolic manifolds in 3-dimensional topology has been revealed through the work of Thurston [T1], [T2], [T3]. In particular, there is the following conjecture.

Conjecture 2.4 (Thurston [T2]). An irreducible 3-manifold $M$ such that $\pi_{1}(M)$ is infinite and has no subgroup isomorphic to $\mathbb{Z} \times \mathbb{Z}$ is hyperbolic.

Thurston has shown that this is true if $M$ is Haken, i.e., is irreducible and contains an incompressible surface [T2], [T3].

(b) $\pi_{1}(M)$ has a subgroup isomorphic to $\mathbb{Z} \times \mathbb{Z}$. In order to discuss this case, we need to recall some facts about Seifert fiber spaces [Se], [Or], [Sco3].

A Seifert fiber space is a 3-manifold $M$ which can be expressed as a disjoint union of circles (fibers) such that each fiber has a fibered solid torus neighborhood, given by identifying the ends of a solid cylinder $D^{2} \times I$ by a rotation through $\frac{2 \pi p}{q},(p, q)=1, p \geq 1$, the fibers coming from the $I$-fibers of $D^{2} \times I$ in the obvious way. If $p \geq 2$, the central core of $D^{2} \times S^{1}$ is a singular fiber of multiplicity $p$. In particular, $M$ has a foliation by circles. Conversely, by [Ep] any foliation of a 3-manifold by circles is of this form.

The quotient space of $M$ obtained by identifying each fiber to a point, is a surface, the base (surface) of the Seifert fibration.

The only reducible Seifert fiber spaces are $S^{1} \times S^{2}$ and $P^{3} \# P^{3}$.

It is convenient to divide the irreducible Seifert fiber spaces into the following classes: those that have Seifert fiberings with

(A) base $S^{2}$ and at most 2 singular fibers;

(B), (C), (D) base $S^{2}$ and 3 singular fibers, of multiplicities $p_{1}, p_{2}, p_{3}$, where $\sum \frac{1}{p_{i}}>1$, $=1$, or $<1$ respectively;

(E) all others.

Remarks. (1) The manifolds in class (A) are $S^{3}$ and the lens spaces. They are precisely the Seifert fiber spaces with finite cyclic $\pi_{1}$. 
The manifolds in class (B) are precisely the Seifert fiber spaces with finite non-cyclic $\pi_{1}$. Note that the possibilities for $\left(p_{1}, p_{2}, p_{3}\right)$ are the Platonic triples $(2,2, n),(2,3,3)$, $(2,3,4)$ and $(2,3,5)$.

The manifolds in classes (A) and (B) are precisely the spherical 3-manifolds.

(2) A Seifert fiber space $M$ is Haken if and only if either $M$ is in class (E) (in which case $M$ contains a vertical incompressible torus), or $M$ is in class (C) or (D) and $H_{1}(M)$ is infinite (in which case $M$ contains a horizontal incompressible surface). In particular, if we say that a 3-manifold is toroidal if it contains an incompressible torus (and atoroidal otherwise), and if we subdivide class $(\mathrm{C})$ into classes $\left(\mathrm{C}^{\prime}\right)$ and $\left(\mathrm{C}^{\prime \prime}\right)$ according to whether $H_{1}(M)$ is finite or infinite, then the toroidal Seifert fiber spaces are precisely those in classes $(\mathrm{E})$ and $\left(\mathrm{C}^{\prime \prime}\right)$ (the latter contain horizontal incompressible tori). We remark that $\left(\mathrm{C}^{\prime}\right)$ is the set of manifolds in $(\mathrm{C})$ that have Nil geometric structures, while $\left(\mathrm{C}^{\prime \prime}\right)$ is the set of manifolds in $(\mathrm{C})$ that have euclidean structures. (See [Sco3] for an excellent account of the eight 3-dimensional geometries.)

For the purposes of this paper, let us call the Seifert fiber spaces in classes (A), (B), (C) and (D) small (as in [BW]), and those in classes $\left(\mathrm{C}^{\prime}\right)$ and (D) exceptional. Thus the exceptional Seifert fiber spaces are precisely the irreducible, atoroidal Seifert fiber spaces with infinite $\pi_{1}$. Note that although such a manifold $M$ is atoroidal, $\pi_{1}(M)$ has a subgroup isomorphic to $\mathbb{Z} \times \mathbb{Z}$.

We are now ready to state the theorem that holds in this subcase (b).

THEOREM 2.5. Let $M$ be an irreducible 3-manifold such that $\pi_{1}(M)$ has a subgroup isomorphic to $\mathbb{Z} \times \mathbb{Z}$. Then $M$ is either toroidal or an exceptional Seifert fiber space.

This theorem is the culmination of work by several people. For $M$ Haken it was announced by Waldhausen [Wa], and a proof given by Feustel [F1], [F2]. If $M$ is nonHaken, Scott showed [Sco1] that the hypothesis of the theorem implies that $\pi_{1}(M)$ has an infinite cyclic normal subgroup $Z$. Mess showed [Me] that $\pi_{1}(M) / Z$ is either the fundamental group of a 2-dimensional orbifold or a discrete convergence group. In the first case, another result of Scott [Sco2] implies that $M$ is a Seifert fiber space. Finally, Casson and Jungreis [CJ] and Gabai [Ga3] independently showed that a discrete convergence group is a Fuchsian group, implying that $M$ is a Seifert fiber space in the second case also.

After Theorem 2.5, Thurston's Geometrization Conjecture [T1], [T2], [T3] is equivalent to the conjunction of Conjectures 2.3 and 2.4.

3. Dehn filling. Let $M$ be a compact irreducible 3-manifold with $\partial M$ a torus. Let $r$ be a slope on $\partial M$, that is, the isotopy class of an essential simple closed curve on $\partial M$. The (closed) 3-manifold obtained from $M$ by $r$-Dehn filling is defined to be $M(r)=M \cup V_{r}$, where $V_{r}$ is a solid torus, attached to $M$ along the boundary in such a way that $r$ bounds a disk in $V_{r}$.

If $M$ is a Seifert fiber space (the definition given in Section 2 applies verbatim to manifolds with boundary) then $M(r)$ is a Seifert fiber space unless $r=r_{0}$ is the slope of a fiber, while $M\left(r_{0}\right)$ is a connected sum of lens spaces and copies of $S^{1} \times S^{2}$ [He]. 
If $M$ contains an incompressible torus which is not parallel to $\partial M$, then this torus usually remains incompressible in $M(r)$. In fact the exceptions can be explicitly described, by results of Berge [B1], Gabai [Ga2] and Scharlemann [Sch2].

By Thurston's geometrization theorem for Haken manifolds [T2], [T3], in the remaining case $M$ is hyperbolic, i.e., int $M$ has a complete hyperbolic structure.

Let us generalize the discussion for a moment to allow manifolds with more than one boundary component. We shall say that a (compact, orientable) 3-manifold $M$ is hyperbolic if int $M$ has a complete hyperbolic structure and $M$ is not homeomorphic to $S^{1} \times D^{2}$ or $T^{2} \times I$. Now let $M$ be a hyperbolic 3 -manifold with a torus boundary component $T$ (and possibly other boundary components, which need not be tori). Define $E(M)=E(M, T)=\{r: r$ is a slope on $T$ such that $M(r)$ is not hyperbolic $\}$, the set of exceptional slopes of $M$ (on $T$ ), and let $e(M)=|E(M)|$. The goal is to obtain restrictions on the set $E(M)$.

If $r, s$ are two slopes on $T$, the distance $\Delta(r, s)$ between $r$ and $s$ is defined to be their minimal geometric intersection number. Most of the results we describe will be in the form of upper bounds on $\Delta(r, s)$ for particular classes of exceptional slopes $r$ and $s$.

Note that if $\{\mu, \lambda\}$ is a basis for $H_{1}(T)$, then the slopes on $T$ can be parametrized by $\mathbb{Q} \cup\{1 / 0=\infty\}$, by the correspondence $r \leftrightarrow a / b$, where $[r]=a \mu+b \lambda$. If $s \leftrightarrow c / d$ then $\Delta(r, s)=|a d-b c|$. If $M_{L}=S^{3}-\operatorname{int} N(L)$ is the exterior of a link $L$ in $S^{3}$, then we will always parametrize the slopes on $\partial M_{L}$ in this way by taking $\mu$ and $\lambda$ to be a meridian and longitude for the appropriate component of $L$.

Here are two examples, which we shall have occasion to refer to later.

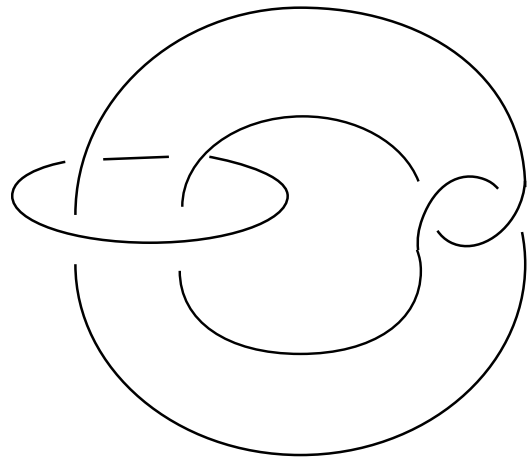

Figure 3.1

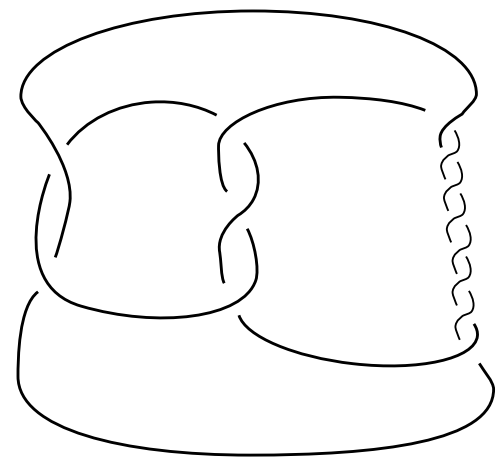

Figure 3.2

ExAmple (1). Let $L$ be the Whitehead link, illustrated in Figure 3.1, and let $W$ be the exterior of $L$. Let $T$ be one of the components of $\partial W$. (Since there is an isotopy of $S^{3}$ interchanging the components of $L$, it is immaterial which component is chosen.) Then it is proved in [NR] that $E(W)=E(W, T)=\{\infty, 0,1,2,3,4\}$. (Note that [NR] works with the mirror-image of $L$, so that our slopes are the negatives of those given there.) Thus $e(W)=6$.

EXAMPLE (2). Let $L^{\prime}$ be the $(-2,3,8)$ pretzel link, also called the Whitehead sister link [BFLW] (see Figure 3.2), and let $W^{\prime}$ be the exterior of $L^{\prime}$. The volumes of $W$ 
and $W^{\prime}$ are equal. Let $T$ be the component of $\partial W^{\prime}$ corresponding to the unknotted component of $L^{\prime}$. Then, using Weeks' computer program snappea, Hodgson and Weeks have shown that $E\left(W^{\prime}\right)=E\left(W^{\prime}, T\right)=\left\{\infty, 2,3, \frac{7}{2}, \frac{11}{3}, 4\right\}$, giving $e\left(W^{\prime}\right)=6$. (Since there is an automorphism of $W^{\prime}$ interchanging its boundary components, there are also six exceptional slopes on the other boundary component.) Note that $\Delta\left(2, \frac{11}{3}\right)=5$.

By Thurston's geometrization theorem for Haken manifolds [T2], [T3], a 3-manifold with non-empty boundary is hyperbolic if and only if it is irreducible, atoroidal (i.e., every incompressible torus is boundary parallel) and not a Seifert fiber space. Hence if a 3-manifold with non-empty boundary is not hyperbolic then it contains an essential surface of non-negative euler characteristic (i.e., a sphere, disk, torus or annulus). By analyzing when such surfaces can be created by two distinct Dehn fillings on a manifold $M$, one can prove the following.

THEOREM 3.1 [Go2]. Let $M$ be a hyperbolic 3-manifold with a torus boundary component $T$ and at least one other boundary component. Then $\Delta(r, s) \leq 5$ for all $r, s \in E(M)$.

Example (2) above shows that this bound is best possible.

It follows from Theorem 3.1 that $e(M) \leq 8$. The examples given above have $e(M)=6$. No example is known with $e(M)>6$ (where $M$ has more than one boundary component).

From now on we will consider the harder case where $\partial M$ consists of a single torus. A fundamental result of Thurston says that $e(M)$ is finite [T1], [T2].

Note that, assuming the Geometrization Conjecture (i.e., Conjectures 2.3 and 2.4), if $M(r)$ fails to be hyperbolic then it is either reducible, toroidal, or a small Seifert fiber space. For the results we shall describe, it turns out that we can ignore Conjecture 2.3, but in view of the fact that Conjecture 2.4 is still open it is necessary to work with a slightly different definition of an exceptional slope. Namely, let us say that a closed 3-manifold $M$ is hyperbolike if it is irreducible, and $\pi_{1}(M)$ is infinite and has no subgroup isomorphic to $\mathbb{Z} \times \mathbb{Z}$. Thus hyperbolic 3 -manifolds are hyperbolike, and the converse assertion is Conjecture 2.4.

Let RED, CYC, FIN, TOR and ESFS be the set of (closed) 3-manifolds that are reducible, have (finite) cyclic $\pi_{1}$, have finite non-cyclic $\pi_{1}$, are toroidal, or are exceptional Seifert fiber spaces, respectively. Then, by Theorem 2.5, a 3-manifold is not hyperbolike if and only if it belongs to RED $\cup$ CYC $\cup$ FIN $\cup$ TOR $\cup$ ESFS.

Now define $E^{\prime}(M)=\{r: M(r)$ is not hyperbolike $\}$, and $e^{\prime}(M)=\left|E^{\prime}(M)\right|$. Thus $E^{\prime}(M) \subset E(M)$, with conjectured equality.

At the moment the best universal bounds on $E^{\prime}(M)$ are the following.

TheOREm 3.2 (Bleiler-Hodgson [BH1]). $e^{\prime}(M) \leq 24$, and $\Delta(r, s) \leq 22$ for all $r, s \in$ $E^{\prime}(M)$.

The proof of this theorem uses the " $2 \pi$ " theorem of Gromov and Thurston, together with a result of Adams on cusp volume [A1], to show that, for $r$ outside a set of slopes of the kind described, $M(r)$ has a Riemannian metric of negative sectional curvature (and is therefore hyperbolike).

Before giving more detailed bounds, we describe some examples of manifolds $M$ with relatively large values of $e(M)$ and $\Delta(r, s), r, s \in E(M)$. These will all be of the form 
$W(r)$ or $W^{\prime}(r)$, obtained by Dehn filling on one of the boundary components of the exterior of the Whitehead or Whitehead sister link.

First consider $W$; recall that $E(W)=\{\infty, 0,1,2,3,4\}$. For these exceptional slopes, the discussion in [HMW, Proposition 3] shows that $W(\infty) \cong S^{1} \times D^{2}, W(1), W(2)$ and $W(3)$ are Seifert fiber spaces with base $D^{2}$ and two singular fibers, $W(0)$ contains a non-separating torus, and $W(4)$ contains a Klein bottle. It follows that for any slope $r$, if $M=W(r)$ then $M(\infty)$ is a lens space or $S^{3}$ or $S^{1} \times S^{2}$, each of $M(1), M(2)$ and $M(3)$ is either a small Seifert fiber space or a connected sum of two lens spaces, and $M(0)$ and $M(4)$ contain a non-separating torus and Klein bottle, respectively. Hence $E(M) \supset$ $\{\infty, 0,1,2,3,4\}$. This gives infinitely many hyperbolic manifolds $M$ with boundary a torus having $e(M) \geq 6$.

Similarly, we get infinitely many manifolds with $e(M) \geq 6$ from the Whitehead sister exterior.

EXAmple (3). Let $K$ be the figure eight knot. Then $M_{K}=W(-1)$. Note that since $K$ is amphicheiral, $M_{K}(-r) \cong-M_{K}(r)$, and in fact $E\left(M_{K}\right)=\{\infty, 0, \pm 1, \pm 2, \pm 3, \pm 4\}$. Thus $e\left(M_{K}\right)=10$. Specifically, $M_{K}(\infty) \cong S^{3}, M_{K}(0), M_{K}( \pm 4) \in$ TOR, and $M_{K}( \pm 1)$, $M_{K}( \pm 2), M_{K}( \pm 3) \in$ ESFS.

Example (4). $M=W(5)$. Here $E(M)=\left\{\infty, 0,1, \frac{4}{3}, \frac{3}{2}, 2,3,4\right\}$. Thus $e(M)=8$. The nature of the manifolds $M(r), r \in E(M)$, and the distances $\Delta(r, s), r, s \in E(M)$, are shown in Figure 3.3. (Unlabelled distances are 1.)

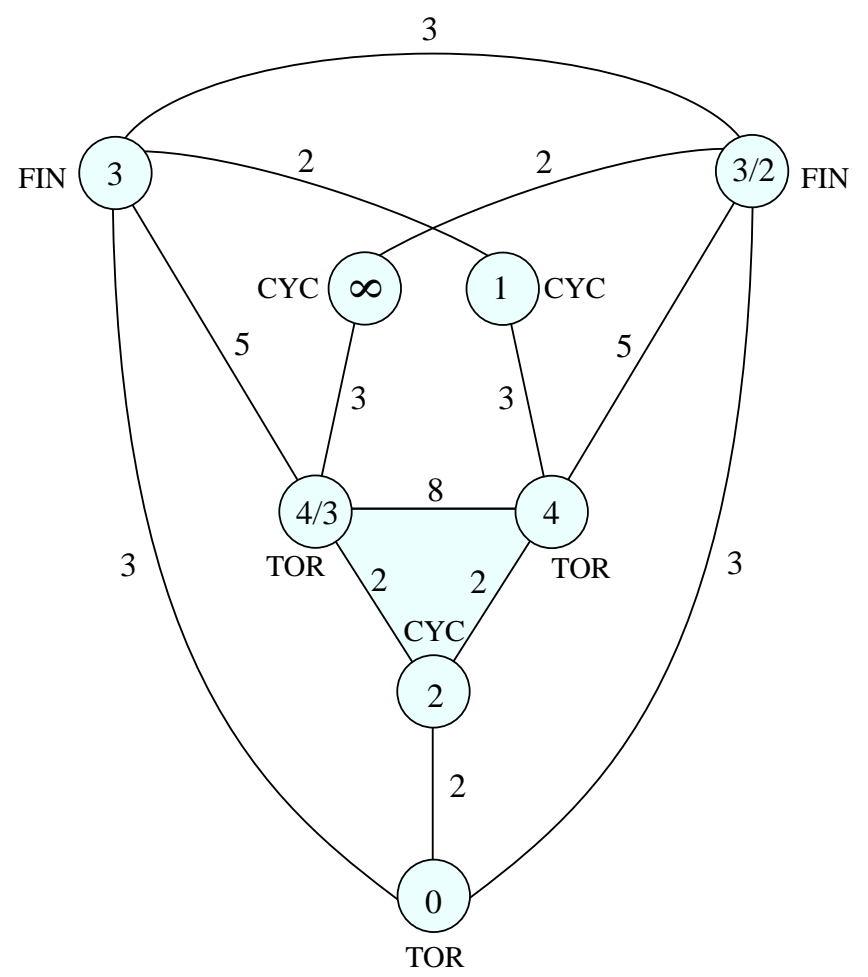

Figure 3.3 
Example (5). $M=W(-2)$. Here $E(M)=\{\infty, 0, \pm 1, \pm 2,3,4\}$, so $e(M)=8$. Specifically, $M(\infty) \in \mathrm{CYC}, M( \pm 1), M(2), M(3) \in \mathrm{ESFS}, M(0), M(-2), M(4) \in \mathrm{TOR}$.

Example $(6)$. Let $K$ be the $(-2,3,7)$ pretzel knot. Then $E\left(M_{K}\right)=\left\{\infty, 16,17,18, \frac{37}{2}\right.$, $19,20\}$, giving $e\left(M_{K}\right)=7$, as shown in Figure 3.4.

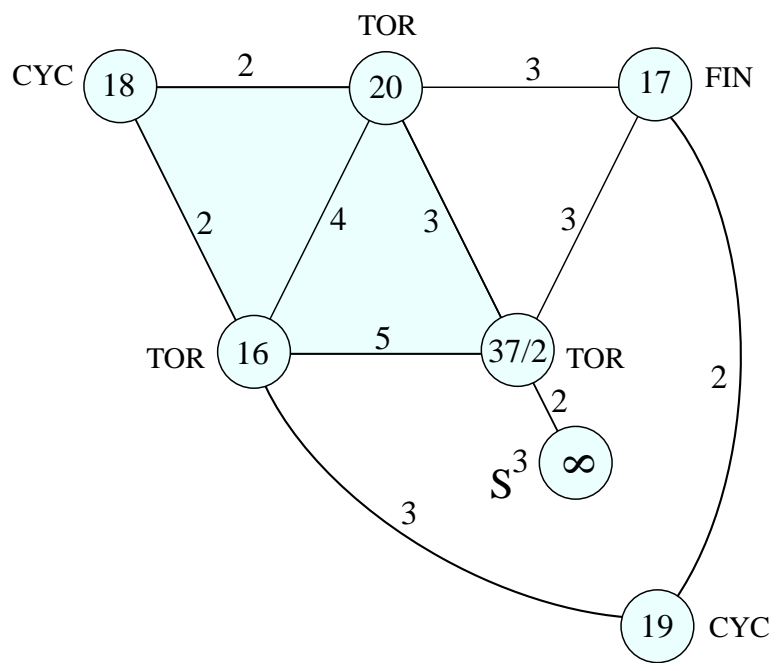

Figure 3.4

Example (7). $M=W(6)$. Then $M(\infty) \in \mathrm{CYC}, M(1) \in \mathrm{RED}, M(2) \in \mathrm{FIN}, M(3) \in$ ESFS, $M(0), M(4) \in$ TOR.

Example (8). Let $M_{n}=W\left(\frac{1}{n}\right),|n|>1$. Then $M_{n}$ is the exterior of the $n$-twist knot in $S^{3}$. We have $E\left(M_{n}\right)=\{\infty, 0,1,2,3,4\}$.

Remarks. (1) The determination of the exceptional fillings on the figure eight knot exterior (Example (3)) is due to Thurston [T1].

(2) Example (4) is the figure eight sister manifold, studied in [We]. It is also homeomorphic to $W^{\prime}(5)$. It has the same volume as the figure eight knot complement. It is proved in [BPZ] that $E(M) \subset\left\{\infty, 0,1, \frac{4}{3}, \frac{3}{2}, 2,3,4\right\}$. The fact that $\frac{4}{3}$ and $\frac{3}{2} \in E(M)$ has been verified by Hodgson and Weeks [HW] using snappea.

(3) For Example (5), the proof that the exceptional slopes are as stated is given in [BPZ].

(4) Example (6) first appears (somewhat disguised) in [FS]. It turns out that $M_{K}=$ $W^{\prime}(1)$. The determination of the exceptional slopes has been done by Hodgson and Weeks [HW] using snappea. For a discussion of the exceptional fillings, see [Eu2].

(5) Example (7) is given in [BZ2].

(6) In Example (8), the fact that there are no other exceptional slopes is proved in $[\mathrm{BW}]$, using the Orbifold Geometrization Conjecture and snappea.

Using snappea, Hodgson has compiled a considerable amount of data concerning Dehn fillings on hyperbolic 3-manifolds which can be decomposed into at most five ideal 
tetrahedra. In addition to Example (3), with $e(M)=10$, Examples (4) and (5), with $e(M)=8$, and Example $(6)$, with $e(M)=7$, he has found seven other manifolds $M$, all but one of the form $W(r)$, with $e(M)=7$. (Recall that there are infinitely many $M$ with $e(M) \geq 6$.) In view of this data it is tempting to believe that these eleven manifolds are the only ones with $e(M) \geq 7$. In particular, the following is a natural conjecture, which serves as a useful touchstone for results in this area.

CONJECTURE 3.3. Let $M$ be a hyperbolic 3-manifold with boundary a torus. Then $e(M) \leq 8$ unless $M$ is the figure eight knot exterior.

It does not seem too unrealistic to expect that the bounds described below can be refined to at least prove this conjecture with $e(M)$ replaced by $e^{\prime}(M)$.

With the above examples in mind, we now summarize what is known about the possible distances between exceptional slopes.

If $\mathcal{C}_{1}$ and $\mathcal{C}_{2}$ are classes of non-hyperbolike 3 -manifolds, let $\Delta\left(\mathcal{C}_{1}, \mathcal{C}_{2}\right)=\max \left\{\Delta\left(r_{1}, r_{2}\right)\right.$ : $M\left(r_{i}\right) \in \mathcal{C}_{i}, i=1,2$, for some hyperbolic 3 -manifold $M$ with $\partial M$ a torus $\}$. The following table gives upper bounds for $\Delta\left(\mathcal{C}_{1}, \mathcal{C}_{2}\right)$ for the classes $S^{3}\left(=\left\{S^{3}\right\}\right)$, RED, CYC, FIN and TOR.

\begin{tabular}{|c|c|c|c|c|c|}
\hline & $S^{3}$ & RED & CYC & FIN & TOR \\
\hline$S^{3}$ & $0^{*}$ & 1 & $1^{*}$ & 2 & $2^{*}$ \\
\hline RED & & $1^{*}$ & $1^{*}$ & $?$ & $3^{*}$ \\
\hline $\mathrm{CYC}$ & & & $1^{*}$ & $2^{*}$ & $?$ \\
\hline $\mathrm{FIN}$ & & & & 5 & $?$ \\
\hline $\mathrm{TOR}$ & & & & & $8^{*}$ \\
\hline
\end{tabular}

Table 3.1

Remarks. (1) References for these bounds are: $\Delta\left(S^{3}, S^{3}\right)$ [GLu2]; $\Delta\left(S^{3}, \mathrm{RED}\right)$ [GLu1]; $\Delta\left(S^{3}, \mathrm{CYC}\right)$ and $\Delta(\mathrm{CYC}, \mathrm{CYC})[\mathrm{CGLS}] ; \Delta\left(S^{3}, \mathrm{FIN}\right)$ and $\Delta(\mathrm{CYC}, \mathrm{FIN})[\mathrm{BZ} 1]$; $\Delta\left(S^{3}, \mathrm{TOR}\right)[\mathrm{GLu} 4] ; \Delta(\mathrm{RED}, \mathrm{RED})[\mathrm{GLu} 3]$, [BZ2]; $\Delta(\mathrm{RED}, \mathrm{CYC})[\mathrm{BZ} 2] ; \Delta(\mathrm{RED}, \mathrm{TOR})$ [Oh1], [Wu2]; $\Delta$ (FIN, FIN) [BZ1]; $\Delta$ (TOR, TOR) [Go2].

(2) Those bounds marked with a ${ }^{*}$ are best possible. Examples showing this are as follows.

Example (7) shows that $\Delta(\mathrm{RED}, \mathrm{CYC})=1$ and $\Delta(\mathrm{RED}, \mathrm{TOR})=3$.

Example (6) shows that $\Delta\left(S^{3}, \mathrm{CYC}\right)=1, \Delta\left(S^{3}, \mathrm{TOR}\right)=2, \Delta(\mathrm{CYC}, \mathrm{CYC})=1$ and $\Delta(\mathrm{CYC}, \mathrm{FIN})=2$.

Example (3) (or (4)) shows that $\Delta($ TOR, TOR) $=8$.

Finally, an example is given in [GLi] which shows that $\Delta(\mathrm{RED}, \mathrm{RED})=1$. (The manifold $M$ described there has an additional torus boundary component, after capping off the 2-sphere boundary components with 3-balls. However, one can show that doing an appropriate Dehn filling on this boundary component gives a hyperbolic manifold with a single boundary component which still has two reducible fillings at distance 1.) 
(3) The conjectured upper bound for $\Delta\left(S^{3}, \mathrm{RED}\right)$ is 0 (see Conjecture 4.1 below).

Example (7) shows that $\Delta(\mathrm{RED}, \mathrm{FIN}) \geq 1$.

Example (6) shows that $\Delta\left(S^{3}, \mathrm{FIN}\right) \geq 1$.

Example (4) shows that $\Delta($ CYC, TOR $) \geq 3, \Delta($ FIN, FIN $) \geq 3$, and $\Delta($ FIN, TOR $) \geq 5$.

Since no worse examples are known, one expects that these are all equalities.

(4) Regarding $\Delta$ (RED, FIN), Boyer and Zhang have shown [BZ2] that if $M\left(r_{1}\right) \in$ RED and $M\left(r_{2}\right) \in$ FIN then $\Delta\left(r_{1}, r_{2}\right) \leq 5$ unless $M\left(r_{1}\right) \cong P^{3} \# P^{3}$ and $\pi_{1}\left(M\left(r_{2}\right)\right)$ is a $D$-type or $Q$-type group (see [BZ1]).

Also, Oh has shown [Oh2] that if $M\left(r_{1}\right) \in \mathrm{RED}$ and $M\left(r_{2}\right)$ is a Seifert fiber space with base $S^{2}$ of type $(2,2, n)$ then $\Delta\left(r_{1}, r_{2}\right) \leq 3$.

(5) Regarding $\Delta$ (TOR, TOR), it is shown in [Go2] that if $M\left(r_{1}\right)$ and $M\left(r_{2}\right) \in$ TOR, then $\Delta\left(r_{1}, r_{2}\right) \leq 5$ unless $M=W(-1), W(5), W(5 / 2)$ or $W(-2)$. In these cases, there exist $r_{1}, r_{2}$ with $M\left(r_{1}\right)$ and $M\left(r_{2}\right) \in$ TOR such that $\Delta\left(r_{1}, r_{2}\right)=8,8,7$ and 6 respectively.

We may summarize the situation by saying that the above table gives quite good bounds on the distances between exceptional slopes, except for those that correspond to exceptional Seifert fiber spaces. In particular, the lack of good bounds in this case is the main obstruction to proving (the weak form of) Conjecture 3.3. Note that Example (3) shows that $\Delta\left(\right.$ ESFS,$\left.S^{3}\right) \geq 1, \Delta($ ESFS, ESFS $) \geq 6$, and $\Delta($ ESFS, TOR $) \geq 7$. Example $(7)$ shows that $\Delta$ (ESFS, RED) $\geq 2$. Also, there are examples given by Hodgson and Weeks in $[\mathrm{HW}]$ showing that $\Delta(\mathrm{ESFS}, \mathrm{CYC}) \geq 2$ and $\Delta(\mathrm{ESFS}, \mathrm{FIN}) \geq 2$.

The data suggests the following.

Conjecture 3.4. Let $M$ be a hyperbolic 3-manifold with boundary a torus. If $M$ is not homeomorphic to $W(-1), W(5), W(5 / 2)$ or $W(-2)$, then $\Delta(r, s) \leq 5$ for all $r, s \in E(M)$.

This would imply Conjecture 3.3 .

The known manifolds $M$ with $e(M)$ large are all "small." For example, the Whitehead and Whitehead sister link exteriors $W$ and $W^{\prime}$ are conjecturally the manifolds with the lowest volume among all hyperbolic 3 -manifolds with two cusps (see $[\mathrm{CHW}]$ ). Again, $\operatorname{vol} W(-1)=\operatorname{vol} W(5), \operatorname{vol} W(5 / 2)$, and $\operatorname{vol} W(-2)$ are conjecturally the lowest, second lowest, and third lowest volumes of hyperbolic 3-manifolds with one cusp [CHW].

The Whitehead and Whitehead sister links have tunnel number 1 , so each of $W$ and $W^{\prime}$ can be obtained from a genus 2 handlebody by adding a 2-handle, in other words, the Heegaard genus $g(W)=g\left(W^{\prime}\right)=2$. It follows that $g(W(r)) \leq 2$ for all $r$, and $g(W(r)(s)) \leq 2$ for all $r$ and $s$ (and similarly for $W^{\prime}$ ). On the other hand, since the Heegaard genus of a small Seifert fiber space is at most 2, one might expect that a manifold $M$ with several exceptional Dehn fillings of this kind should have $g(M)=2$. In this context it is interesting to note that Adams has shown [A2] that if $g(M)>2$ then in Theorem 3.2 the bounds of 24 and 22 can be replaced by 16 .

For information on the behavior of Heegaard genus under Dehn filling see [MR].

4. Knots in the 3-sphere. In this section we make some remarks about the special case of Dehn filling on the exterior $M_{K}$ of a hyperbolic knot $K$ in $S^{3}$. For further information see the very nice survey article $[\mathrm{Lu}]$. In this case we shall abbreviate $M_{K}(r)$ to 
$K(r), E^{\prime}\left(M_{K}\right)$ to $E^{\prime}(K)$, etc. Note that $\infty \in E^{\prime}(K)$, since $K(\infty) \cong S^{3}$; hence $e^{\prime}(K) \geq 1$. However, as we will discuss below, a picture is emerging which suggests that it might eventually be possible to give a complete "list" of all knots $K$ with $e^{\prime}(K)>1$.

So let $K$ be a hyperbolic knot in $S^{3}$ and suppose that $r \in E^{\prime}(K)$. We shall always assume that $r \neq \infty$.

First consider the case when $K(r)$ is reducible. The Cabling Conjecture [GS] asserts that (allowing $K$ to be arbitrary for the moment) $K(r)$ is reducible for some $r$ if and only if $K$ is a cable knot. Since this is known to be true if $K$ is a satellite knot [Sch2], the conjecture is equivalent to the following statement for hyperbolic knots.

Conjecture 4.1. $K(r)$ is always irreducible.

This is known to be true for several classes of knots; for example, alternating knots [MT], strongly invertible knots [Eu1], symmetric knots [HS], arborescent knots [Wu1], and knots of bridge number at most 4 [Hof]. It is true if $r=0$ [Ga1]; more generally, if $K(r)$ is reducible then it must have a lens space summand [GLu2]. It is also known that if $K(r)$ is reducible then $\Delta(r, \infty)=1$ (see Table 3.1). However, a general proof of the Cabling Conjecture continues to be elusive. It is one of the most interesting open questions about Dehn surgery on knots.

Regarding the case when $K(r)$ is toroidal, here we have $\Delta(r, \infty) \leq 2$ (see Table 3.1). There are many examples with $\Delta(r, \infty)=1$ (see [Pat] for the case of alternating knots), and it may be too ambitious to try to describe them all.

Eudave-Muñoz has described infinitely many examples with $\Delta(r, \infty)=2$ [Eu2], and asks if these are the only such examples. So we have

Conjecture 4.2. If $K(r)$ is toroidal for some $r$ with $\Delta(r, \infty)=2$, then $K$ is a Eudave-Muñoz knot.

Eudave-Muñoz' examples all have the following properties: $K$ is strongly invertible and has tunnel number 1 , and if $K_{r}$ denotes the core of the attached solid torus $V_{r}$ in $K(r)$, then there is an incompressible torus $T$ in $K(r)$ such that $\left|K_{r} \cap T\right|=2$, separating $K(r)$ into two Seifert fiber spaces with base $D^{2}$ and two singular fibers. The following theorem might therefore be regarded as evidence in favor of Conjecture 4.2.

Theorem 4.3 [GLu4], [GLu5]. If $K(r)$ is toroidal with $\Delta(r, \infty)=2$ then

(1) $K$ is strongly invertible;

(2) $K$ has tunnel number at most 2;

(3) there is an incompressible torus $T$ in $K(r)$ such that $\left|K_{r} \cap T\right|=2$;

(4) one component of $K(r)$ cut along $T$ is a Seifert fiber space with base $D^{2}$ and two singular fibers.

Concerning condition (3), Eudave-Muñoz [Eu3] has recently constructed examples where $K(r)$ is toroidal, with $\Delta(r, \infty)=1$, such that the incompressible torus $T$ in $K(r)$ with $\left|K_{r} \cap T\right|$ minimal has $\left|K_{r} \cap T\right|=4$.

Turning to the case when $\pi_{1}(K(r))$ is finite cyclic, we have the bounds given in Table 3.1. If $\pi_{1}(K(r))$ is trivial, then it is known that $K(r)$ cannot be $S^{3}$ (see Table 3.1), 
but the following old Property P Conjecture [BM] is still open for hyperbolic knots (it is true for non-hyperbolic knots by [Se] and [Ga2] (see also [Sch1])).

Conjecture 4.4. $\pi_{1}(K(r)) \neq 1$.

It is a nice challenge to find a proof of this before someone proves the Poincaré Conjecture.

For $\pi_{1}(K(r))$ finite cyclic and non-trivial, Example (6) in Section 3 shows that the relevant bounds given in Table 3.1 are best possible.

In this context there is a beautiful construction due to Berge [B2], which goes as follows: Let $X$ be a handlebody of genus 2 standardly embedded in $S^{3}$, and let $X^{\prime}=$ $\overline{S^{3}-X}$ be the complementary handlebody. Let $K$ be a non-trivial knot in $S^{3}$ which lies on $\partial X\left(=\partial X^{\prime}\right)$ in such a way that the manifold $X(K)$ obtained by attaching a 2-handle to $X$ along $K$ is a solid torus, and similarly for $X^{\prime}$. Then if $r$ is the slope of the framing of $K$ induced from $\partial X$, it is easy to see that $K(r)=X(K) \cup X^{\prime}(K)$, and hence is a lens space (it cannot be $S^{1} \times S^{2}$ by [Ga1], nor $S^{3}$ by [GLu2]). Berge has given a complete list of such knots $K$, and has suggested

ConjeCture 4.5. If $K(r)$ is a lens space then $(K, r)$ arises from Berge's construction.

Dean [D] has studied a variant of Berge's construction in which $K$ is as before except that one now insists that $X^{\prime}(K)$, instead of being a solid torus, is a Seifert fiber space (with base $D^{2}$ and two singular fibers). Then $K(r)$ (if irreducible) will be a small Seifert fiber space.

Conjecture 4.6. If $K(r)$ is a Seifert fiber space other than a lens space then $(K, r)$ arises from Dean's construction.

Conjectures 4.5 and 4.6 would imply

Conjecture 4.7. If $K(r)$ is a Seifert fiber space then it is a small Seifert fiber space.

In the direction of Conjecture 4.7, Boyer and Zhang have shown [BZ2] that if $K(r)$ is a Haken Seifert fiber space then $\Delta(r, \infty)=1$.

Conjectures 4.5 and 4.6 also imply

ConjeCture 4.8. If $K(r)$ is a Seifert fiber space then $\Delta(r, \infty)=1$.

Recall from Table 3.1 that it is known that $\Delta(r, \infty)=1$ if $K(r) \in \mathrm{CYC}$, and that $\Delta(r, \infty) \leq 2$ if $K(r) \in$ FIN.

Roughly speaking, the Cabling Conjecture, and Conjectures 4.2, 4.5 and 4.6, all say that the existence of the exceptional filling in question has a relatively simple topological explanation.

We note that Eudave-Muñoz knots, Berge knots, and Dean knots all have tunnel number 1. On the other hand, Eudave-Muñoz and Luecke (unpublished) have constructed knots $K$ with arbitrarily high tunnel number such that $K(r)$ is toroidal for some $r$ with $\Delta(r, \infty)=1$.

Finally, we remark that many interesting results on Dehn filling have been obtained using the theory of laminations. We refer the reader to [Ga4] for a nice account of these, and additional references. 


\section{References}

[A1] C. Adams, The noncompact hyperbolic 3-manifold of minimal volume, Proc. Amer. Math. Soc. 100 (1987), 601-606.

[A2] C. Adams, Unknotting tunnels in hyperbolic 3-manifolds, Math. Ann. 302 (1995), 177-195.

[B1] J. Berge, The knots in $D^{2} \times S^{1}$ which have nontrivial Dehn surgeries that yield $D^{2} \times S^{1}$, Topology and its Applications 39 (1991), 1-19.

[B2] J. Berge, Some knots with surgeries yielding lens spaces, unpublished manuscript.

[BPZ] S. Betley, J. H. Przytycki and T. Zukowski, Hyperbolic structures on Dehn filling of some punctured-torus bundles over $S^{1}$, Kobe J. Math 3 (1986), 117-147.

[BM] R. H. Bing and J. M. Martin, Cubes with knotted holes, Trans. Amer. Math. Soc. 155 (1971), 217-231.

[BH1] S. Bleiler and C. Hodgson, Spherical space forms and Dehn surgery, Knots 90, Proceedings of the International Conference on Knot Theory and Related Topics (A. Kawauchi, ed.), Osaka (Japan), de Gruyter, Berlin, New York, 1992, 425-433.

[BH2] S. Bleiler and C. Hodgson, Spherical space forms and Dehn filling, Topology 35 (1996), 809-833.

[BZ1] S. Boyer and X. Zhang, Finite Dehn surgery on knots, preprint.

[BZ2] S. Boyer and X. Zhang, The semi-norm and Dehn filling, preprint.

[BW] M. Brittenham and Y.-Q. Wu, The classification of Dehn surgeries on 2-bridge knots, preprint.

[BFLW] A. M. Brunner, M. L. Frame, Y. W. Lee, and N. J. Wielenberg, Classifying torsion-free subgroups of the Picard group, Trans. Amer. Math. Soc. 282 (1984), 205-235.

[CHW] P. J. Callahan, M. V. Hildebrand and J. R. Weeks, A census of cusped hyperbolic 3-manifolds, preprint.

[CJ] A. Casson and D. Jungreis, Convergence groups and Seifert fibered 3-manifolds, Invent. Math. 118 (1994), 441-456.

[CGLS] M. Culler, C. McA. Gordon, J. Luecke and P. B. Shalen, Dehn surgery on knots, Ann. of Math. 125 (1987), 237-300.

[D] J. Dean, Ph.D. thesis, University of Texas at Austin, 1996.

[Ep] D. B. A. Epstein, Periodic flows on three-manifolds, Ann. of Math. 95 (1972), 66-82.

[Eu1] M. Eudave-Muñoz, Band sums of links which yield composite links. The cabling conjecture for strongly invertible knots, Trans. Amer. Math. Soc. 330 (1992), 463-501.

[Eu2] M. Eudave-Muñoz, Non-hyperbolic manifolds obtained by Dehn surgery on hyperbolic knots, Proceedings of the Georgia International Topology Conference (1993) (to appear).

[Eu3] M. Eudave-Muñoz, 4-punctured tori on the exterior of knots, preprint.

[EM] B. Evans and J. Maxwell, Quaternion actions on $S^{3}$, Amer. J. Math. 101 (1979), 1123-1130.

[F1] C. D. Feustel, On the torus theorem and its applications, Trans. Amer. Math. Soc. 217 (1976), 1-43.

[F2] C. D. Feustel, On the torus theorem for closed 3-manifolds, Trans. Amer. Math. Soc. 217 (1976), 45-57.

[FS] R. Fintushel and R. Stern, Constructing lens spaces by surgery on knots, Math. Z. 175 (1980), 33-51. 
[Ga1] D. Gabai, Foliations and the topology of 3-manifolds, III, J. Diff. Geom. 26 (1987), 479-536.

[Ga2] D. Gabai, Surgery on knots in solid tori, Topology 28 (1989), 1-6.

[Ga3] D. Gabai, Convergence groups are Fuchsian groups, Ann. of Math. 136 (1992), $447-510$

[Ga4] D. Gabai, Eight problems in the geometric theory of foliations and laminations on 3-manifolds, preprint.

[GS] F. González-Acuña and H. Short, Knot surgery and primeness, Math. Proc. Camb. Phil. Soc. 99 (1986), 89-102.

[Go1] C. McA. Gordon, Dehn surgery on knots, Proceedings of the International Congress of Mathematicians, Kyoto, 1990, Springer-Verlag, Tokyo, 1991, pp. 631-642

[Go2] C. McA. Gordon, Boundary slopes of punctured tori in 3-manifolds, Trans. Amer. Math. Soc. (to appear).

[GLi] C. McA. Gordon and R. A. Litherland, Incompressible planar surfaces in 3manifolds, Topology and its Applications 18 (1984), 121-144.

[GLu1] C. McA. Gordon and J. Luecke, Only integral Dehn surgeries can yield reducible manifolds, Math. Proc. Camb. Phil. Soc. 102 (1987), 94-101.

[GLu2] C. McA. Gordon and J. Luecke, Knots are determined by their complements, J. Amer. Math. Soc. 2 (1989), 371-415.

[GLu3] C. McA. Gordon and J. Luecke, Reducible manifolds and Dehn surgery, Topology 35 (1996), 385-409.

[GLu4] C. McA. Gordon and J. Luecke, Dehn surgeries on knots creating essential tori, I, Communications in Analysis and Geometry 3 (1995), 597-644.

[GLu5] C. McA. Gordon and J. Luecke, Dehn surgeries on knots creating essential tori, II, Comm. Anal. Geom. (to appear).

[HS] C. Hayashi and K. Shimokawa, Symmetric knots satisfy the cabling conjecture, preprint.

[He] W. Heil, Elementary surgery on Seifert fiber spaces, Yokohama Math. J. 22 (1974), 135-139.

[HMW] C. D. Hodgson, G. R. Meyerhoff, and J. R. Weeks, Surgeries on the Whitehead link yield geometrically similar manifolds, Topology '90 (B. Apanasov, W.D. Neumann, A.W. Reid and L. Siebenmann, eds.) de Gruyter, Berlin, 1992, pp. 195-206.

[HW] C. D. Hodgson and J. R. Weeks, A census of closed hyperbolic 3-manifolds, in preparation.

[Hof] J. Hoffman, Ph.D. thesis, The University of Texas at Austin, 1995.

[Hop] H. Hopf, Zum Clifford-Kleinschen Raumproblem, Math. Ann. 95 (1925), 313-319.

[K] H. Kneser, Geschlossene Flächen in dreidimensionale Mannigfaltigkeiten, Jahresber. Deutsch. Math.-Verein. 38 (1929), 248-260.

[Le] R. Lee, Semicharacteristic classes, Topology 12 (1973), 183-199.

[Li] G. R. Livesay, Fixed point free involutions on the 3-sphere, Ann. of Math. 72 (1960), 603-611.

[Lu] J. Luecke, Dehn surgery on knots in the 3-sphere, Proceedings of the International Congress of Mathematicians, Zürich, 1994, Birkhäuser Verlag, Switzerland, 1995, pp. 585-594.

[MT] W. Menasco and M. Thistlethwaite, Surfaces with boundary in alternating knot exteriors, J. Reine Angew. Math. 426 (1992), 47-65. 
[Me] G. Mess, Centers of 3-manifold groups and groups which are coarse quasiisometric to planes, preprint.

[Mi1] J. Milnor, Groups which act on $S^{n}$ without fixed points, Amer. J. Math. 79 (1957), 623-630.

[Mi2] J. Milnor, A unique factorization theorem for 3-manifolds, Amer. J. Math. 84 (1962), 1-7.

[MR] Y. Moriah and H. Rubinstein, Heegaard structures of negatively curved 3-manifolds, preprint.

[My] R. Myers, Free involutions on lens spaces, Topology 20 (1981), 313-318.

[NR] W. D. Neumann and A. W. Reid, Arithmetic of hyperbolic manifolds, Topology '90, (B. Apanasov, W. D. Neumann, A. W. Reid and L. Siebenmann, eds.), de Gruyter, Berlin, 1992, pp. 273-310.

[Oh1] S. Oh, Reducible and toroidal 3-manifolds obtained by Dehn filling, Topology and its Applications, (to appear).

[Oh2] S. Oh, Dehn filling, reducible 3-manifolds, and Klein bottles, preprint.

[Or] P. Orlik, Seifert manifolds, Lecture Notes in Mathematics, vol. 291, Springer, Berlin, 1972.

[Pap] C. D. Papakyriakopoulos, On Dehn's lemma and the asphericity of knots, Ann. of Math. 66 (1957), 1-26.

[Pat] R. Patton, Incompressible punctured tori in the complements of alternating knots, Math. Ann. 301 (1995), 1-22.

[Ric] P. M. Rice, Free actions of $Z_{4}$ on $S^{3}$, Duke Math. J. 36 (1969), 749-751.

[Rit] G. X. Ritter, Free $Z_{8}$ actions on $S^{3}$, Trans. Amer. Math. Soc. 181 (1973), 195-212.

[Ru] J. H. Rubinstein, Free actions of some finite groups on $S^{3}$. I, Math. Ann. 240 (1979), 165-175.

[Sch1] M. Scharlemann, Sutured manifolds and generalized Thurston norms, J. Diff. Geom. 29 (1989), 557-614.

[Sch2] M. Scharlemann, Producing reducible 3-manifolds by surgery on a knot, Topology 29 (1990), 481-500.

[Sco1] P. Scott, A new proof of the annulus and torus theorems, Amer. J. Math. 102 (1980), 241-277.

[Sco2] P. Scott, There are no fake Seifert fibre spaces with infinite $\pi_{1}$, Ann. of Math. 117 (1983), 35-70.

[Sco3] P. Scott, The geometries of 3-manifolds, Bull. London Math. Soc. 15 (1983), 401-487.

[Se] H. Seifert, Topologie dreidimensionaler gefaserter Räume, Acta Math. 60 (1932), 147-238.

[TS] W. Threlfall and H. Seifert, Topologische Untersuchung der Discontinuitätsbereiche endlicher Bewegungsgruppen des dreidimensionalen sphärischen Raumes, I, Math. Ann. 104 (1930), 1-70; II, 107 (1932), 543-586.

[T1] W. P. Thurston, The Geometry and Topology of 3-manifolds, Princeton University, 1978.

[T2] W. P. Thurston, Three dimensional manifolds, Kleinian groups and hyperbolic geometry, Bull. Amer. Math. Soc. 6 (1982), 357-381.

[T3] W. P. Thurston, Hyperbolic structures on 3-manifolds, I: Deformation of acylindrical manifolds, Ann. of Math. 124 (1986), 203-246. 
[Wa] F. Waldhausen, On the determination of some bounded 3-manifolds by their fundamental groups alone, Proc. Inter. Sym. Topology, Hercy-Novi, Yugoslavia, 1968; Beograd, 1969, pp. 331-332.

[WS] C. Weber and H. Seifert, Die beiden Dodekaederräume, Math. Z. 37 (1933), 237-253.

[We] J. R. Weeks, Hyperbolic structures on three-manifolds, Ph.D. thesis, Princeton University, 1985.

[Wh] J. H. C. Whitehead, On 2-spheres in 3-manifolds, Bull. Amer. Math. Soc. 64 (1958), 161-166.

[Wu1] Y.-Q. Wu, Dehn surgery on arborescent knots, J. Diff. Geom. 43 (1996), 171-197.

[Wu2] Y.-Q. Wu, Dehn fillings producing reducible manifold and toroidal manifold, preprint. 